\title{
The First Original Copy And The Role Of Blockchain In The Reproduction Of Cultural
}

\section{Heritage}

\author{
Eugene Ch'ng \\ eugene.chng@nottingham.edu.cn \\ NVIDIA Joint-Lab on Mixed Reality \\ University of Nottingham Ningbo China \\ 199 Taikang East Road, Ningbo 315100, China
}

\begin{abstract}
The First Original Copy refers to any first true 3D facsimile of digitally reproduced physical object. The notion of a copy being the first and original implies that it is unique and therefore the approach used for managing rights and ownership influences its value. Whilst virtual goods traded within virtual worlds are subject to rules and policies, the production of digital objects in the real world does not have a mechanism from which rarity and uniqueness can be guaranteed. Digital copies are subject to further copying and thus, the value of even an exact copy can never be perceived to be equivalent to its original. Through what means can we imbue 3D reproductions of cultural objects with value that is at least asymptotic to their originals? There may be a candidate solution. Discussed in this paper is a possible approach for resolving a long-term issue related to authenticity, ownership, perpetuity and the quantitative tracking of value associated with 3D copies. Blockchains essentially bring the systemic management of virtual objects within virtual worlds into the real world. This forum article examines the candidate solution by answering the questions above, and discusses the issues associated with the concept of the First Original Copy.
\end{abstract}


Keywords: Blockchain, authenticity, first original copy, ownership, photogrammetry, digital heritage

\section{Introduction}

The value of material culture is founded on both its physical and intangible properties. These are aesthetics and the authenticity of the object, its condition and quality, and the intangible values associated with the object, such as its symbolism and provenance, past rituals and historical development, all of which contributed to the uniqueness of the object. Due to such uniqueness, cultural relics are immutable, irreproducible and as a consequent irreplaceable. This said the materiality of it is unfortunately ephemeral. The value attributed to the value of the object is its singularity, for there can exist only one original object, the rest are copies made from it. When we reproduce a digital facsimile of the original object, the quality of the $3 \mathrm{D}$ copy whilst retaining the true appearance of the original, is suddenly inverted. The digital copy becomes mutable, reproducible and, if stored in the right medium, can become a perpetual record. However, when digitized, the authenticity and value of the copy of the original appears to have been lost, for the copy can now be edited, reproduced and distributed at little costs. Decades of 3D reproductions have yielded no solution to such a problem.

\section{The Background of the Discourse}

UNESCO defines Cultural heritage as "the legacy of physical artefacts and intangible attributes of a group or society that are inherited from past generations, maintained in the present and bestowed for the benefit of future generations." Specific to this article, tangible heritage includes "buildings and historic places, monuments, artifacts, etc., which are considered worthy of preservation for the future. These include objects significant to the archaeology, architecture, science or technology of a specific culture.” (UNESCO, 2018). 
The definition and scope of heritage is defined and redefined by many institutions and has a well-established history (Ahmad, 2006; Vecco, 2010). What we are dealing with here is the reproduction of $3 \mathrm{D}$ digital copies from original physical objects.

The candidate solution to a global issue I aim to provide here is as a result of a yearlong discourse on the \#ReACHDialogue, a UNESCO endorsed initiative spearheaded by the Victoria \& Albert Museum in partnership with the Peri Charitable Foundation. ReACH (Reproduction of Art and Cultural Heritage) was initiated to review Henry Cole's 1867 convention (Cole, 1867) signed by the prominent people of his age "for promoting universally reproductions of works of art for the benefit of museums of all countries" for public instruction. Reproductions from 150 years ago were "Casts, Electrotypes, Photographs, and other processes, without the slightest damage to the originals." The ReACH Initiative has since reviewed and redrafted Henry Cole's convention in light of the $4^{\text {th }}$ Industrial Revolution, in order "to explore how our imperilled cultural heritage can be preserved in our digital era of 3D printing, ultra-high resolution and drone technology, and to debate the creative opportunities that copying these works offers a global audience." (ReACHDeclaration, 2018). I contributed as a global consultant to ReACH in four talks on sustainability issues, while conceptualising the idea of the First Original Copy discussed here and the identification of six types of reproduction (Figure 1).

1. "Sustainable Strategies for the Digital Production and Sharing of Artworks and Cultural Heritage” ReACH Roundtable Discussion, Beijing Palace Museum, Beijing, China. 30 November 2017

2. "Sustainable Sharing in the Digital Age" V\&A ReACH Conference - finalisation of the new Convention for Reproduction of Art and Cultural Heritage. Victoria and Albert Museum, London, UK. 8 December 2017

3. "The Challenges of Digital Storage" V\&A ReACH Technical Policy Round-Table. 
Department of Culture and Tourism, Abu Dhabi, UAE. 23-24 April 2018

4. "Sustainable Digital Heritage” UNESCO Headquarters, Book Launch and Conference. Paris, France. 22 June 2018.

Henry Cole's 1867 convention dealt mainly with the first two types of reproduction, from physical to physical, such as plaster casts, and from physical to analogue, such as photography. Challenges appear when the $4^{\text {th }}$ Industrial Revolution (Schwab, 2017) increasingly blurs the line between the physical and the digital. The reproduction of cultural heritage exists within such a revolution with regards to the four additional types of reproduction.

My final speech at UNESCO addressing the member states highlighted the need for a democratised and sustainable solution to 3D digital copies. Having worked within the digital heritage discipline for almost two decades, particularly with the reproduction of heritage objects, monuments and sites over many Virtual Reality projects, I have found the greatest challenge to be the issue of sustainability of storage and access across both time and space. As such, a global initiative such as $\mathrm{ReACH}$ has become a platform providing individuals, groups and institutions facing similar challenges with a communal basis for resource sharing and the development of best practices. The book 'Copy Culture: Sharing in the Age of Digital Reproduction' (Cormier, 2018) launched at the UNESCO ReACH conference provides the basis for future community-led development.

Figure 1 here

Copies such as plaster casts made in the past century are physical, they are therefore unique in their own rights. Many of the earlier physical copies have become invaluable today. Stored and exhibited at the V\&A's Cast Court is the earliest example, the cast of Trajan's Column taken from the original $1^{\text {st }}$ Century AD monument. The value of copies is seen when the real Trajan's Column lost surface detail due to pollution and acid rain whilst the original 
copy remained intact. A digital copy is now being made of that cast, resulting in a first digital copy of an original physical copy. Here we see that early physical copies are still unique, and their value can be said to be asymptotic to the original. Digital reproductions have no such value, and will unlikely grow in value over time without some digital mechanisms securing access rights. Once reproduced, they are formulae of representations embedded within silicon and voltage, reconstructed and displayed on digital screens when demanded. Digital copies once obtained, are subject to change, they are transmutable. The act of copying takes no effort and distributing copies takes up only the bandwidth proportional to the byte size of the digital copy. Whilst the value is in the permanency and accessibility of such objects in both time and space, there is no secure record of the First Original Copy. Scarcity is not in the nature of digital copies and therefore value cannot be guaranteed nor maintained. There are in fact no mechanisms for authenticating digital copies. Once copied and distributed, there can be no distinction between the first copy and its subsequent copies. Unless there is institutional endorsement, anyone owning any copies could claim rights and ownership to them from copies distributed worldwide. Whilst institutions possess the means to resolve such a problem via online publishing, digital copies of cultural heritage made by individuals and groups in impoverished and marginalised societies is likely to be taken advantage of.

Recent advances in photogrammetry techniques have made such technologies accessible and pervasive that its widespread use for value creation and economic profit is just a matter of time, particularly when both the East and the West are marrying technology with culture (see Culture is Digital executive summary report (Hancock, 2018) and China's Cultural Technology Innovation Plan (Hancock, 2018)). 3D digital recording and subsequent copying have become commonplace. In an interview with Financial Times (Brown, 2016), Brendan Cormier of the V\&A responded that "We need to get over the stigma of the copy, ...we should no longer be asking, 'Should we or should we not copy?' The question is, 'What 
should we be copying and for what purposes?' And that inevitably brings you to political questions." Reproductions has a very real currency, Aguerre and Cormier stated that "The proliferation of images of works of art, in fact, has become a significant driver for going to museums - the opportunity to see the original, finally, after having seen the reproduction so many times over.” (Cormier, 2018). Whilst copying has become a positive activity, digital copying is in urgent need of a mechanism for securing ownership.

The scope of this article does not delve into issues related to cultural heritage as 'property' under law, which has been discussed at length (Prott \& O'Keefe, 1992), although in the periphery of the issues discussed, rights and ownership of 3D facsimiles are implied. The article focuses on the First Original Copy of original objects produced worldwide over the past decades when 3D capture became a possibility, and how blockchain technology can be a solution to many issues faced by the global community of copiers. It is felt that such a topic is best discussed as a forum paper within PRESENCE as the journal encompasses both the art and science of virtual environments in an age where virtual, intangible objects have generated much profit from both virtual worlds and the creative industry.

\section{The Value of Original Objects and its Virtual Copies}

Why are cultural heritage objects valuable when they are made from physical materials that are in abundance? These sorts of questions, much like historical debates from the Institutional Theory of Art (Dickie, 1969), are difficult to answer as the value of a heritage object depended on many factors. Andy Warhol's Brillo Boxes (1964) is an example. One cannot argue that the materiality of it decides the value of it, for the same cardboard boxes endorsed as Art within a gallery are no different from cardboard boxes sold in stores. The 'Fountain' (1917) by Marcel Duchamp is no different from the urinals installed in the Gents' in the early 1900s. The value of a certain relic made of stone can potentially outweighs the value of an object made of gold. Like Art, the first condition of an object is that "it must first 
be an artefact" (ibid). An artifact is "an object made by a human being, typically one of cultural or historical interest" as defined by the Oxford Dictionary. The fact that a historical artefact has value is not a credit to the object itself, but to the intangible properties embedded within the object through past human activities. These properties imbuing the physical object with aura could be a ritual associated with the object, the stories behind it, the context in which the object was created, its archaism and, at times, purely the rarity of it. Whilst I do not wish to delve into a purely theoretical perspective in dealing with value, they are nevertheless useful for setting the context for our discussion.

The theory of value has a long historical discourse beginning from Plato in the "Republic" (402E) (Plato, 2005) from which intrinsic and instrumental value were debated. Cultural heritage is regarded as having intrinsic value, and is an end in itself. In contrast with instrumental value associated with moral and nonmoral goods, intrinsic value does not fluctuate but grow over time. Cultural artifacts therefore, have existence or sentimental value (Krutilla, 1967), a value system referring to the satisfaction people may obtain from knowing that a heritage site or monument exists. Such value appeals to individuals or communities having particular attachment to a relic, which can be attributed to the object's social, aesthetic or historical value (Beck, 1995). Artifacts also have bequest value ("The Heritage Dividend methodology: measuring the impact of heritage projects," 2005), a value associated with the satisfaction obtained from knowing that the object is preserved for the future generation. The reason for existence or bequest value is often associated with an artifact's aesthetic value (Goldman, 2018; Tsugawa, 1968), which is a personal, individual appreciation or judgment of the positive value an object may bring. Such judgments are necessarily not a moral or empirical judgment. Whilst cultural relics may not be owned by an individual and does not have personal value, which provides some benefits to an individual so much so that it influences the behavior and choices made by the individual (Rokeach, 1968, 1973), they have 
cultural value in that they capture the shared value, shared expectations and the collective understanding within a society. Cultural value is increased when an object is institutionalized. When institutional endorsement is strong, in circumstances, individuals or a community may be willing to pay, i.e., option value (Weisbrod, 1964), for preserving artifacts which are of no instrumental value in the present nor in the future in the public domain. The intrinsic value of cultural heritage therefore, captures what is worthwhile, important and good to an individual or collectively as a society.

In terms of function, cultural heritage does have instrumental value. They simply are economic profits gained as a result of the financial contributions from heritage tourism, from heritage products created as a result of high value relics, and the broader context of socioeconomic, and educational benefits to communities.

Unlike goods of hedonistic or use value, various analysis of the economics of cultural heritage and the physical assets and products associated with them suggests that there are no market value from which exchanges would be made (Aplin, 2004; Hutter \& Rizzo, 1997; Mourato \& Mazzanti, 2002). This includes various methods using contingency valuation first applied in the 1990s (Mitchell \& Carson, 1989). Contingency valuation refers to measures of the willingness to pay for the maintenance and/or accessibility of a cultural resource. It has been applied in many scenarios, for example, the willingness to pay for cultural services and the willingness to pay (Bravi, Scarpa, \& Sirchia, 2002; Cuccia \& Signorello, 2000), anthropogenic service value and hedonic service value (Calver \& Page, 2013), travel costs (Bedate, Herrero, \& Sanz, 2004), social benefits (Salazar \& Marques, 2005) and a volume of work on environmental valuation (Navrud \& Ready, 2002), from which cultural heritage are thus valued for their economic properties. These cultural heritage goods have no well-defined market processes of buying and selling, and the lack of a pricing system attached to the goods. Instead, the willingness to pay depended upon many factors, including the observable 
The article has been accepted for publication in PRESENCE 27(1). Citeable as: Eugene Ch'ng (2019) The First Original Copy And The Role Of Blockchain In The Reproduction Of Cultural Heritage, PRESENCE 27(1).

and non-observable characteristics of the goods.

All tangible cultural heritage objects are subject to the value systems stated above. Will digital artefacts ever hold the same value? Are concepts of value in the real world transferrable to the virtual world? We live in a world where physical materials worked upon by past cultures become unique, and where the difficulty of replicating a physical work with manual labor makes a relic rare and therefore precious. In the digital world however, the moment copies are made, they are immediately subjected to further copying. Digitisation therefore breaks the scarcity principle. Digital replicas appear to not have the same intrinsic value in our immediate perception, even though they may have the exact appearances. Will 3D digital copies of cultural heritage carry Walter Benjamin's concept of aura (Benjamin, 2008) is a topic worth a good discussion. If one has insights, one will see that digital facsimiles are a permanent representation whilst the original objects can be subjected to entropy, they erode over time and are at risk of anthropogenic hazards. In time, the replicas may even assume the role of the original and perhaps supersede it (Ch'ng, 2013), as in the case of the cast of Trajan's column. Are digital copies as valuable as its originals? There is no straightforward answer, at least not until the digital has completely mediated our physical world, and digital contents are consumed as fervently as physical objects, and when signs and symbols replace and simulate our reality. The precession of simulacra may be at work already (Baudrillard, 1994). We may be enlightened to see that apart from the tangibility of digital copies, there are no differences in their appearances. Furthermore, physical artifacts are not as accessible as their digital replicas. One thing is clear, whilst the intrinsic value of a copy is not equivalent to its original, the instrumental value of the copy is significantly far greater. Here we see the differences of value between the original and its copy - the former has intrinsic value while the latter has instrumental value. If the two are put together, the value will perhaps become greater than the original. This is the reason why digital copying has 
become prevalent in our society. We can also begin to understand why digital transformation has become a mandatory process within the Arts and Humanities. The question is whether we can bestow intrinsic value to digital copies, at least for the first copy of the original?

Let us briefly look at the observed trend of the economy of virtual goods within virtual worlds and computer games as arguments for the value of digital copies. Newzoo's Global Games Market Report forecasts that 2.3 billion gamers globally will spend US\$137.9 billion on games in 2018 , an increase of $+13.3 \%$ from 2017 , and that digital game revenues will take $91 \%$ of the global market worth US $\$ 125.3$ billion. This includes a large proportion of virtual goods bought and traded within the virtual and gaming worlds. An earlier press release from Second Life reported a US\$3 billion in virtual goods transactions over the course of its lifetime (VirtualGoodsSecondLife, 2013). If virtual goods are perceived as real within virtual reality, and bought with real-world currencies, then perhaps the population of digital users are ripe for the consumption of digital copies of cultural artifacts, if such copies can be made unique, rare and authentic. Virtual goods exist solely within virtual worlds, within a system of economy where policies can be implemented and rules are strictly governed. Such virtual and gaming policies ensure the rarity and uniqueness of objects within the system. Digital reproduction of cultural heritage exists outside of such a system. They are stored in local computers and can be freely distributed. Digital copies need a real-world, democratised system and policy to dictate its use and value. If implemented, such a system and policy will bring many other benefits which we will discuss in the next section. It is believed that once the issue of the First Original Copy is resolved, the rest of the issues will be resolved as well.

\section{Problems with Virtual Copies}

Cultural heritage objects are non-moral goods. But due to their individual uniqueness and rarity, they can be rivalrous and excludible even in the public goods domain. Some heritage sites are rivalrous, they are congestible due to the limits of the physical space. 
The article has been accepted for publication in PRESENCE 27(1). Citeable as: Eugene Ch'ng (2019) The First Original Copy And The Role Of Blockchain In The Reproduction Of Cultural Heritage, PRESENCE 27(1).

For example, the West Minster cathedral may only contain so many persons within its spaces, leaving others out in the queue. Digital copies are nonrivalrous and not congestible. The nonexcludibility of it presents a problem where value is concerned.

\section{The Issue of the First Original Copy}

Digital cultural heritage objects if distributed through the right channels, (e.g., Sketchfab.org) can become nonrivalrous. Congestibility will no longer be an issue if demands are managed via distributed servers. However, the need for distributed servers does mean that multiple copies are stored in server caches distributed across large geographical locations. Whilst users may perceive that there is only one true copy on the website, there are actually multiple copies on the Web. Digital copies offered to global audiences are often surrogates. They are not true facsimiles as they are intended to be streamed and viewable on the Web. As such, they tended to carry a small file size and as a consequent, the need for heavy decimation of the geometry. These 3D models while downloadable, will not be of much use and value to subject domain experts as their lack of surface details have rendered them noninterpretable. As such, surrogates are pointers to the original copy and therefore good only for public appreciation. Herein lies the problem. Publishing the First Original Copy as a true facsimile on the Web is risky and no owners of any models will yield to such a thought, as there are no methods for securing and tracing the true copy. Copies copied from the First Original Copy are no different in nature and appearance from the original copy and therefore, all copies can be claimed as the First Original Copy. This complicated statement does reflect the complexity of managing copies. Digital watermarking can be introduced, but additional digital watermarks can be inserted into the second copy with both parties claiming ownership to it. The fact is that there is presently no digital rights management for 3D models. 
The article has been accepted for publication in PRESENCE 27(1). Citeable as: Eugene Ch'ng (2019) The First Original Copy And The Role Of Blockchain In The Reproduction Of Cultural Heritage, PRESENCE 27(1).

True facsimiles can also be sold under a nondistributable, nontransferable contract but if the model is somehow stolen, many copies can be made, often out of a malicious intent or as an act of mischief. Copies produced by large institutions can be protected if the right to scan is provided by the institution itself, and if the original, such as the cast of Trajan's Column is a well-known, identifiable model. However, there are presently many unaccountable original objects in the world, the majority of them are unknown, and unprotected by cultural institutions and governing bodies. Many of these items are inheritances residing in the homes of the present generation. Others are circulated in the antique market. There are also archaeological items yet to be discovered. I am certain that there will be, at some point in time, a First Original Copy of any of all the growing number of relics in the world created by present cultures. There is no way of authenticating the First Original Copy as part of an ownership that can be accredited to the author.

We have to this point discussed the issue of the First Original Copy as true facsimiles, and surrogates as mere pointers to them. In summary, surrogates are made accessible as low quality models, whereas true facsimiles are the real issue as they cannot be made accessible due to the lack of the management of rights and ownership, the availability of methods of authentication, and appropriate strategies for storage, traceability and permanency of access. These issues are all related and linked to the concept of the First Original Copy. They will be discussed in detail in the subsequent sections.

\section{Identification and Authenticity}

The ability to identify a First Original Copy has implications for the intrinsic and instrumental value of digital objects in the immediate future and for years to come. There may be a descriptive formula which can ascertain the value of an original digital copy. Here is the first attempt - the value of a true original copy is directly proportional to the quality of 
the model multiplied by the intrinsic value of the original, if and only if the First Original Copy can be identified and authenticated.

The ability to identify and authenticate a model as belonging to a reputable source has serious implications for the prevention of misuse, for the crediting of the creator, and the securing of rights and ownership of the original copy. There are presently no means of identifying or distinguishing between the First Original Copy and subsequent copies, and therefore authenticating an original copy is still not possible.

\section{Model Security and Immutability}

The intention of publishing a true facsimile implies that the owner of the first copy is prepared to allow the model to be used as is, decimated and used as a surrogate, or reused as a derivative work. There may also be cases where the First Original Copy is edited in a subtle way for an arbitrary purpose, making it indistinguishable from the original copy. Without a mechanism for securing a model and recording it in place, the copy of the original can be subtly changed and recorded as another original copy. The mutability of a file system embedding the representational information of a 3D model is unquestionable. At present, metadata within a file system storing data can be edited, including the timestamp.

\section{Permanency and Breadth of Access}

How we store our data has already decided how they can be accessed both now and in the future. Permanency of access implies that any copy stored must be made accessible now and in the future. Breadth of access is making copies available to anyone intending to make use of them, without the models being subject to sanctions by unreasonable authorities, thus Net Neutrality is an important principle to follow. Present practices for distributing models are that they are stored in servers owned by groups or corporations, and as such the permanency of such services is questionable as they are subject to corporate sustainability and direction. The LOCKSS principle ('Lots of Copies Keep Stuff Safe') can be practiced, but they do not 
The article has been accepted for publication in PRESENCE 27(1). Citeable as: Eugene Ch'ng (2019) The First Original Copy And The Role Of Blockchain In The Reproduction Of Cultural Heritage, PRESENCE 27(1).

resolve the other issues mentioned in this section.

\section{Accountability, Traceability and Demand Value}

Files embedding original copies can be downloaded, distributed and stored within the local folders of a broad base of users. Once files are downloaded, they can be further copied at a local level, from the source hard disk to the hard disks of other users, they can also be sent via email or any of the social media messenger services available today all without the need for accountability. Once the files are local to an individual, they can also become inaccessible. The traceability of the copy is also an issue. Physical copies can be traced as they are passed from an agent to the next. Digital copies are difficult to trace once downloaded. The importance of traceability must not be underestimated, for they can provide a means for measuring demand value over time and across space, which necessarily includes the traceability of further copies and derivative works, and for the crediting of the source of the model for market value.

\section{Blockchain As a Solution to the First Original Copy}

What is blockchain technology and how can blockchains solve the issues mentioned in the previous section? In essence, a blockchain is a distributed ledger technology. It decentralizes the ledger and allows digital information to be distributed in the network. The digital information being distributed becomes unalterable, they cannot be changed or copied (Tapscott \& Tapscott, 2016). Once digital information cannot be copied and claimed as the original, value can be generated. Blockchain is democratic and secure, and can be a distributed digital rights management technology for copies reproduced from cultural heritage. From the looks of it, blockchain technology essentially brings the systemic management of virtual objects within virtual worlds into the real world.

In a blockchain, there is no intermediary and not the need for one. Trust is peer-to-peer and all transactions are verified by peers. There is no central database owned by any 
individual, nor auditing done by centralized intermediaries. The blockchain continually grows, with each block appended to the previous block. The information on the previous block is used with the information in the current block to create the new block. The distributed ledger is append-only, and therefore, altering or deleting the previous block, or earlier blocks is impossible. In order to change an older block, i.e., add a transaction to an old block, one will have to revalidate all blocks between the older block and the current block. This essentially means that attempts at changing a block requires that you change all the linked blocks, which requires that all the nodes in the network verify all the blocks. A single node will never have the computing resource to verify the chain of blocks, against all the nodes and the growing number of nodes working on the blockchain. The security and permanency of blockchain technology is a solution to authenticating the ownership and identity of the First Original Copy.

\section{A Decentralised Record of Original Copies}

Blockchain technology is the solution to identifying and validating the first original copies reproduced from cultural heritage objects, as well as the further creation of surrogate copies, all recorded as transactions in the blockchain. Copies made from cultural artifacts can have both intrinsic and instrumental value if they are put within a distributed and democratized system, much like the multi-billion dollar economy of virtual goods are commodities within virtual worlds.

Let us explore how the First Original Copy can be created using blockchain technology, and how it can work for the sustainable reproduction, storage and access of cultural heritage:

1. The steward, the first owner records the 3D data of the original physical object together with its metadata and paradata. The 3D model can be digitally watermarked at the point of creation. The data and all associated information, such as the timestamp and owner detail become composite information for 
generating a unique key using Cryptographic Hash Algorithms (e.g., SHA256).

The digital copy now becomes a candidate First Original Copy.

2. The owner requests a transaction for the work, broadcasted to the blockchain's peer-to-peer network composed of nodes. The nodes are computers owned by other owners or users of the blockchain. This can be done immediately, upon completion of the recording of the original object, without going through an intermediary.

3. Next, the forgers in the network of nodes validate the transaction and the owner (the person requesting a transaction) automatically by consensus algorithms.

4. The transaction is verified in combination with other transactions to create a new block of data. The block gets connected to the previous block, adding to the chain of blocks in the blockchain. The transaction is now completed and added to the existing chain of blocks. The First Original Copy is credited to the 'wallet' of the steward.

5. The steward and the forgers are rewarded with tokens for the work done. The First Original Copy is now a permanent record.

The blockchain is immutable and therefore, the record of the First Original Copy, tied to the chain of blocks is immutable. The first publisher who has access to the information creating the composite information is the owner of the model, stamped and secured in the chain. The unique transaction generated from the composite information is difficult to replicate, as they require the effort exerted in reproducing a 3D copy of the artifact, with the owner having a complementary private key.

\section{Consensus System for Validating Copies}

The blockchain network uses consensus algorithms to verify the legitimacy of a transaction used by miners to obtain rewards for their work in performing the verification. In 
cryptocurrencies such as Bitcoin, mining uses Proof of Work (PoW) to verify transactions within each block for validity, and the most efficient miners, i.e., those with the greatest computing hardware gets rewarded if they solve a mathematical puzzle within each block. The mathematical problem cannot be solved in any other way apart from brute force. When a miner finds the right solution to a block, it announces the solution to the whole network, and gets rewarded. The issue with the PoW system is that it consumes extremely large computing resources, and as a result is expensive in in terms of energy consumption (Lilic, 2015). PoW is also flawed, as it will become increasingly centralized where miners or a pool of miners having the greatest computing resource controls the majority of the network. Energy efficiency can be increased with renewable energy; however, the centralization of this route is not suitable for our cause.

Another consensus called Proof of Stake (PoS) is used for validating transactions but does so without mining. PoS uses very little energy, is secure and extremely fast. Miners are called forgers in PoS and the tokens they own determine their stakes. Those who have more stakes become more trustworthy as the tokens they own and use as stakes make them more riskaverse. In the PoS consensus system, forgers deposit their tokens into the system to get the opportunity to validate the block. If a forger has more tokens, the stakes will be higher and subsequently their chances of creating and signing the block become higher. Forgers with the highest stake get rewarded with more tokens (transaction fees) and their value to the network becomes higher. In such a system good stewards become 'richer'. At any given time, more stewards and forgers are coming into the network and contributing copies, contributing to a healthy ecology of value creation for digital heritage.

We can now see that the PoS system is more suited to our cause. Tokens can be generated by being good stewards contributing to the production of digital copies as new transactions in the blockchain. The more original copies one produces, the more stakes one has. Whilst 
forgers need not necessarily be stewards, they can generate tokens by becoming forgers within the network, and, sooner or later, the forgers will themselves increase their stakes by becoming stewards. Moderation strategy can be introduced for tackling derivative copies via forking for example. Demands for true original copies can also elevate the copies up in the value chain.

There are blockchain implementations which combine consensus systems such as PoW and PoS, but PoW should be avoided at all cost for cultural heritage. For our sake, PoW needs not be used as minting is not necessary for noncurrency transactions. I believe that original copies are currencies in themselves with future exchange value. We do however, want to encourage more and more forgers to maintain the network, and for reproducing original copies of cultural heritage. Proof of Activity (PoA) may be used later as an incentive for maintaining forger value in the network.

\section{Value within a Blockchain of Copies}

How are values created for digital copies using blockchain technology? Blockchains can solve issues with the First Original Copy of cultural heritage artifacts, allowing for rights, ownership and identity to be secured and authenticated. It can also encourage the production of copies by stewards, with rewards for forgers who maintain the network. Original copies put into the blockchain network is value in itself.

Within a blockchain, value is created and transactions recorded for good stewards who contribute original copies of artifacts. Stewards can be motivated to share their original copies with confidence. The sharing of cultural heritage is encouraged as the exposure and distribution of artefacts bridge knowledge gaps and creates awareness for heritage. Forgers also gain value by validating transactions and creating blocks and therefore increases their stakes. Forgers who are also stewards will greatly increase value if effort is put into reproducing cultural heritage and registering them as transactions within the blockchain. As 
original copies can be downloaded on demand without going through an intermediary, agent fees are exempted and therefore both producers and consumers of copies gain value. Fees paid for using copies are recorded as transactions, and stored permanently in the blockchain. Through the transactions, demands are recorded and the use of original copies can be enumerated and traced. Through blockchains as a distributed system, the issue of the maintenance of storage and permanency of access is also resolved.

\section{Conclusion}

In this article, the argument for the use of blockchain for securing the First Original Copy was discussed. Blockchain technology can bring the systemic management of virtual goods and value creation used in virtual worlds into the real world.

The solution to the fundamental need to identify and authenticate digital copies has important implications for the permanent digital documentation of cultural heritage, especially when erosion, damage, neglect and destruction from anthropogenic hazards and conflict threaten our physical cultural heritage. Having the ability to identify and authenticate original copies has a definite positive cascading effect, which can guarantee the security and immutability of true 3D facsimiles. It is a sustainable solution to the issue of the permanency of storage and accessibility, for accountability and traceability, and for the recording of demand and exchange value. The importance of a sustainable and democratic mechanism for creating value within an increasingly digital world therefore cannot be ignored. Through blockchains the instrumental value of digital copies can be greatly increased, as the uniqueness and rarity of copies can be made possible. In such a system, the protection of digital resources created by individuals and marginalised groups can be assured.

The notion of the intrinsic value of heritage objects for an old world where scarcity of physical resources limiting access is acceptable and commonplace. But intrinsic value may not exist in a world where rampant copying serves to distribute original copies far and wide. 
There needs to be a solution for the issue of the First Original Copy reproduced from all artifacts in the world, secured within a system with mechanisms for managing rights and ownership, and for authenticating its use. Such a solution is projected to be important for the creation of instrumental value and the transfer of intrinsic value for cultural heritage in the future. The cast of Trajan's Column had great instrumental value at the time of production in 1864, and not until the original column has lost its magnificence to pollution and acid rain did the original cast copy become important for it retained all surface details of the original column. It is a certainty that the cast copy of Trajan's Column now possesses intrinsic value, transferred from the original monument. The cast copy of Trajan's Column is presently being digitized and it is a matter of time before the original cast copy loses its brilliance, which leaves the first digital copy as the only true copy in the world. Perhaps then will the intrinsic value of the cast copy be transferred to the First Original Copy.

\section{Acknowledgement}

The author wishes to express his appreciation to the Victoria and Albert Museum and the Peri Charitable Foundation for spearheading the ReACH Initiative. Special gratitude to Anaïs Aguerre for leading and coordinating the programme, which connected a global community of thinkers and doers for the future of the reproduction of art and cultural heritage.

\section{References}

Ahmad, Y. (2006). The scope and definitions of heritage: from tangible to intangible. International Journal of Heritage Studies, 12(3), 292-300.

Aplin, G. (2004). The economics of heritage: A study in the political economy of culture in Sicily. Historic Environment, 17(3), 50.

Baudrillard, J. (1994). Simulacra and simulation. University of Michigan press. 
The article has been accepted for publication in PRESENCE 27(1). Citeable as: Eugene Ch'ng (2019) The First Original Copy And The Role Of Blockchain In The Reproduction Of Cultural Heritage, PRESENCE 27(1).

Beck, H. (1995). Social and aesthetic values. Place, 1, 15-18.

Bedate, A., Herrero, L. C., \& Sanz, J. Á. (2004). Economic valuation of the cultural heritage: application to four case studies in Spain. Journal of Cultural Heritage, 5(1), 101-111.

Benjamin, W. (2008). The work of art in the age of its technological reproducibility, and other writings on media. Harvard University Press.

Bravi, M., Scarpa, R., \& Sirchia, G. (2002). 12. Valuing cultural services in Italian museums: a contingent valuation study. Valuing Cultural Heritage: Applying Environmental Valuation Techniques to Historic Buildings, Monuments and Artifacts, 184.

Brown, G. M. (2016). Art in the age of digital reproduction. Retrieved July 4, 2018, from https:/www.ft.com/content/74ffab6e-1b55-11e6-b286-cddde55ca122

Calver, S. J., \& Page, S. J. (2013). Enlightened hedonism: Exploring the relationship of service value, visitor knowledge and interest, to visitor enjoyment at heritage attractions. Tourism Management, 39, 23-36.

Ch'ng, E. (2013). The Mirror Between Two Worlds: 3D Surface Computing Interaction for Digital Objects and Environments. In Digital Media and Technologies for Virtual Artistic Spaces. Hershey, PA: IGI Global. https://doi.org/10.4018/978-1-4666-2961$5 . \operatorname{ch} 013$

Cole, H. (1867). Convention for Promoting Universally Reproductions of Works of Art for the Benefit of Museums of All Countries. Henry Cole 1867 Convention on the Reproduction of Art.

Cormier, B. (2018). Copy Culture: Sharing in the Age of Digital Reproduction. London: V\&A Publishing.

Cuccia, T., \& Signorello, G. (2000). A Contingent Valuation Study of Willingness to Pay for Visiting a City of Art: The Case Study of Noto (Italy).

Dickie, G. (1969). Defining art. American Philosophical Quarterly, 6(3), 253-256. 
The article has been accepted for publication in PRESENCE 27(1). Citeable as: Eugene Ch'ng (2019) The First Original Copy And The Role Of Blockchain In The Reproduction Of Cultural Heritage, PRESENCE 27(1).

Goldman, A. (2018). Aesthetic value. Routledge.

Hancock, M. (2018). Culture is Digital: Executive summary. Department for Digital, Culture, Media and Sport. Retrieved from https://www.gov.uk/government/publications/cultureis-digital/culture-is-digital

Hutter, M., \& Rizzo, I. (1997). Economic perspectives on cultural heritage. Springer.

Krutilla, J. V. (1967). Conservation reconsidered. The American Economic Review, 57(4), $777-786$.

Lilic, J. (2015). Bitcoins Energy Consumption An Unsustainable Protocol That Must Evolve? Retrieved July 4, 2018, from https://blockgeeks.com/bitcoins-energy-consumption/ Mitchell, R. C., \& Carson, R. T. (1989). Using surveys to value public goods: the contingent valuation method. Rff Press.

Mourato, S., \& Mazzanti, M. (2002). Economic valuation of cultural heritage: evidence and prospects. In M. De la Torre \& D. Throsby (Eds.), Assessing the Values of Cultural Heritage. Getty Conservation Institute.

Navrud, S., \& Ready, R. C. (2002). Valuing cultural heritage: Applying environmental valuation techniques to historic buildings, monuments and artifacts. Edward Elgar Publishing.

Plato, c. (2005). From The Republic. In Readings In The Economics Of The Division Of Labor: The Classical Tradition (pp. 43-49). World Scientific.

Prott, L. V, \& O’Keefe, P. J. (1992). ‘Cultural heritage'or ‘cultural property’? International Journal of Cultural Property, 1(2), 307-320.

ReACHDeclaration. (2018). ReACH: Reproduction of Art and Cultural Heritage. Victoria and Albert Museum Publishing. Retrieved from https://www.vam.ac.uk/research/projects/reach-reproduction-of-art-and-culturalheritage\#outputs 
The article has been accepted for publication in PRESENCE 27(1). Citeable as: Eugene Ch'ng (2019) The First

Original Copy And The Role Of Blockchain In The Reproduction Of Cultural Heritage, PRESENCE 27(1).

Rokeach, M. (1968). Beliefs, attitudes and values: A theory of organization and change.

Rokeach, M. (1973). The nature of human values. Free press.

Salazar, S. D. S., \& Marques, J. M. (2005). Valuing cultural heritage: the social benefits of restoring and old Arab tower. Journal of Cultural Heritage, 6(1), 69-77.

Schwab, K. (2017). The fourth industrial revolution. Crown Business.

Tapscott, D., \& Tapscott, A. (2016). Blockchain revolution: how the technology behind bitcoin is changing money, business, and the world. Penguin.

The Heritage Dividend methodology: measuring the impact of heritage projects. (2005). English Heritage: Valuing the Historic Environment, 2.

Tsugawa, A. (1968). The nature of the aesthetic and human values. Art Education, 21(8), 920.

UNESCO. (2018). Tangible Heritage.

Vecco, M. (2010). A definition of cultural heritage: From the tangible to the intangible. Journal of Cultural Heritage, 11(3), 321-324.

VirtualGoodsSecondLife. (2013). INFOGRAPHIC: 10 YEARS OF SECOND LIFE.

Retrieved July 3, 2018, from https://www.lindenlab.com/releases/infographic-10-yearsof-second-life

Weisbrod, B. A. (1964). Collective-consumption services of individual-consumption goods. The Quarterly Journal of Economics, 78(3), 471-477. 

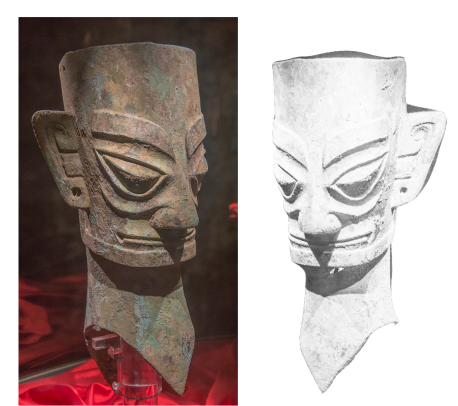

Physical to Physical

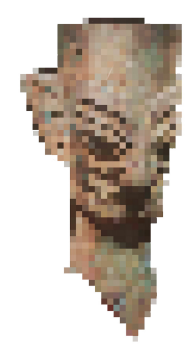

Digital to Digital

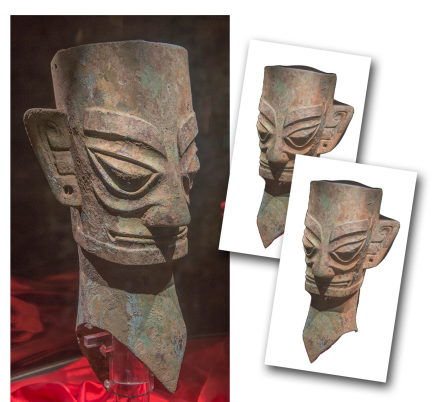

Physical to Analogue

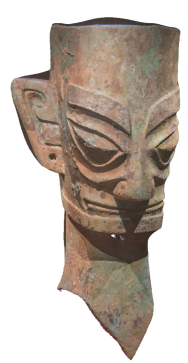

Digital to Physical

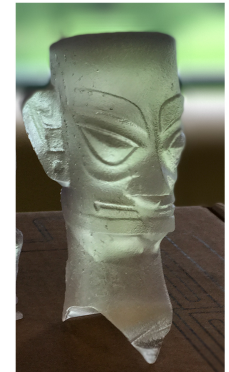

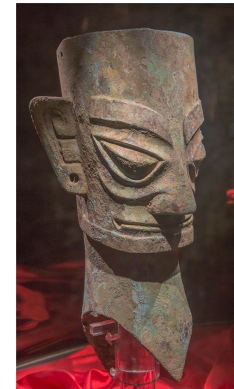

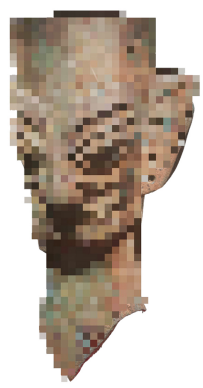

Physical to Digital

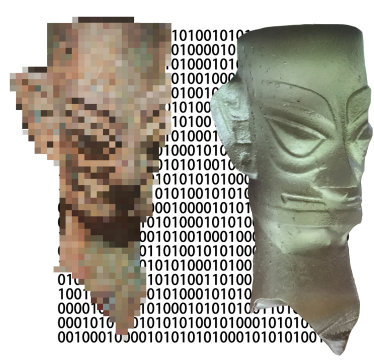

Digital and Physical

Figure 1. The six types of reproduction in the $4^{\text {th }}$ Industrial Revolution. 